\title{
Editorial: Ad Hoc Networking and Emerging Applications
}

\author{
Yifeng Zhou ${ }^{1}$ - Thomas Kunz ${ }^{2}$ - Stefan Fischer ${ }^{3} \cdot$ Zhangdui Zhong $^{4}$
}

Published online: 11 March 2017

(C) Springer Science+Business Media New York 2017

This special issue includes six high quality papers on the topic of ad hoc networking and emerging applications. Among them, three are extended versions of papers recommended by the Technical Program Committee of the 8th EAI International Conference on Ad Hoc Networks (AdHocNets 2016) held in Ottawa, Ontario, Canada on September 26-27, 2016. The other three are selected from an open call. Ad hoc networking covers a variety of network paradigms, such as mobile ad hoc networks (MANETs), wireless sensor networks (WSNs), vehicular networks, underwater networks, airborne networks, home networks, etc., with wide applications in both commercial and military areas. This issue will cover some of the current topics in the field.

The first paper, entitled "Outage probability analysis for energy harvesting cooperative relays in a clustered environment," by Mateen Ashraf, Kyung Geun Lee and Ju

Yifeng Zhou

yifeng.zhou@canada.ca

Thomas Kunz

tkunz@sce.carleton.ca

Stefan Fischer

fischer@itm.uni-luebeck.de

Zhangdui Zhong

zhdzhong@bjtu.edu.cn

1 Communications Research Centre (CRC), 3701 Carling Avenue, Avenue, Box 11490, Station H, Ottawa, ON K2H 8S2, Canada

2 Systems and Computer Engineering, Carleton University, 1125 Colonel By Drive, Ottawa, ON K1S 5B6, Canada

3 Institute of Telematics, University of Lübeck, Ratzeburger Allee 160, 23562 Lübeck, Germany

4 School of Computer and Information Technology, Beijing Jiaotong University, Room 902, Siyuan Building, No. 3 Shangyuancun, Beijing 100044, China
Wook Jang, describes an outage probability analysis of the energy harvesting (EH) decode and forward (DF) cooperative relay network, assuming that the source, the destination and the relays are clustered in a small area. The relays use a power splitting (PS) protocol with adaptive PS ratio for EH. Under a high SNR assumption, approximation of the outage probability and bounds are obtained for two different cooperation schemes, i.e., Selection Corporation and All Relays Cooperation. The bounds and outage probabilities provide guidelines for optimally deploying relays in EH cooperative relay networks.

In the second paper, entitled "Delay constrained relay node placement in wireless sensor networks: A subtree-andmergence-based approach," the authors, Chaofan Ma, Wei Liang and Meng Zheng, discuss the Delay Constrained Relay Node Placement (DCRNP) problem, which searches for a path between a sensor node and the sink using a minimum number of relays under a specified delay constraint. The DCRNP problem is known to be NP-hard. The authors developed a polynomial time Subtree-and-Mergence-based Algorithm (SMA) to approximately solve the DCRNP problem, and provided an analysis of the approximation ratio and the time complexity. They demonstrate using computer simulations that SMA can achieve a performance similar to other existing algorithms in the literature, requiring a significantly smaller number of deployed relays.

The third paper, entitled "A clonal selection algorithm for energy-efficient mobile agent itinerary planning in wireless sensor networks," by Yu-Cheng Chou and Madoka Nakajima, present a new immune inspired algorithm, called the Clonal Selection Algorithm for Multi-agent Itinerary Planning (CSA-MIP), for solving the MIP problem in wireless sensor networks. CSA-MIP has a two-stage evolutionary search procedure, making it resemble a heuristic method that has both global search and local search abilities. The authors show, using computer simulations, that when both stages are activated in CSA-MIP, the diversity in the number of mobile 
agents can be achieved and a higher possibility to obtain solutions of better quality.

The fourth paper, entitled "A multi-pronged approach to adaptive and context aware content dissemination in VANETs," by Joao M. Duarte, Eirini Kalogeiton, Ridha Soua, Gaetano Manzo, Maria Rita Palattella, Antonio Di Maio, Torsten Braun, Thomas Engel, Leandro A. Villas, and Gianluca A. Rizzo, discusses the problem of content dissemination in Vehicular Ad-hoc Networks (VANETs), which has many potential applications in the near future. In this paper, the authors use some reference scenarios to illustrate how to build an innovative adaptive VANET architecture based on Content Centric Networking (CCN), Floating Content (FC) and Software Defined Networking (SDN). As shown by the authors, such an architecture is able to efficiently handle the problems of intermittent connectivity, fluctuating node density and mobility patterns in VANETs, while still being able to provide the desired application performance and an efficient utilization of network resources. The authors also highlight the main open research challenges, and describe possible solutions to improve content dissemination and reduce replication without affecting content availability.

In the fifth paper, entitled "Stigmergic communication for self-organized multi ferry delay tolerant networks," the authors, Mehdi Harounabadi and Andreas Mitschele-Thiel, present an Adaptive On-the-fly Decision maker for Multi Ferry (A-ODMF) delay tolerant networks. A-ODMF assumes a stigmergic communication for coordination among the ferries, and is used to choose the next node to visit by the controlled mobility data ferries. The authors show that A-ODMF is able to adapt its decision functions based on the local observations of a ferry to increase the efficiency of ferry decisions. AODMF also considers the priority of messages in the ferry buffer and delivers high priority messages earlier. Based on simulation results, they showed that A-ODMF outperforms existing on-the-fly approaches in terms of message latency. The authors also study the impact of increasing the number of ferries and increasing the ferry speed on the performance and cost of a message ferry network.

The last paper, entitled "Improving the performance of challenged networks with controlled mobility," is by Laurent Reynaud and Isabelle Guérin-Lassous. The authors investigate an adapted controlled mobility strategy for self-propelling nodes with the objective of efficiently providing network resource to users scattered in a designated area. They designed a virtual force-based controlled mobility scheme, referred to as VFPc, and evaluate its ability to be jointly used with a dual packetforwarding and epidemic routing protocol. They study the delay distribution for synchronous communication traffic by end-users, and show the advantages of VFPc compared to other packet-forwarding and packetreplication schemes.

The guest editors wish to thank all reviewers for their effort in improving the manuscripts. We are also grateful to the Editor-in-Chief, Dr. Imrich Chlamtac for his supportive guidance during the entire process. Last but by no means least, we would like to express our gratitude to Dr. Sara Fruner of Create-Net and Tynie Alfante of Springer for their help in the editorial process. 